\title{
Aprendizagem Baseada em Equipes no ensino odontológico: o que pensam os estudantes?
}

\author{
Emmanoel Matheus de Oliveira Matos*; Ingrid Melo Schüler Arreguy**; Silvia Regina Jamelli***; \\ Fábio Barbosa de Souza****; Elaine Judite de Amorim Carvalho*** \\ * Graduado em Odontologia, UFPE \\ ** Graduanda em Odontologia, UFPE \\ *** Professora Associada, Departamento de Clínica e \\ Odontologia Preventiva, UFPE \\ **** Professor Associado, Departamento de Prótese e Cirurgia \\ Bucomaxilofacial, UFPE
}

Recebido em 16/06/2019. Aprovado em 05/01/2020.

\begin{abstract}
RESUMO
A aprendizagem baseada em equipes (ABE) é um método dinâmico que proporciona um ambiente motivador e cooperativo, com valorização da produção coletiva. O objetivo do presente estudo foi analisar a percepção de estudantes do curso de odontologia de uma universidade brasileira sobre o método ABE. Tratou-se de estudo transversal com amostra de conveniência $(n=83)$, no qual cada aluno respondeu questionário autoaplicável - Instrumento de Avaliação de ABE pelo Estudante. Os dados foram analisados a partir de uma estatística descritiva. Verificou-se uma consistência interna moderada, aceitável (Alfa de Cronbach $=0,736$ ). As médias das respostas obtidas indicaram que os estudantes demonstraram preferência e satisfação quanto ao uso do método ABE, sendo este capaz de despertar maior sentimento de responsabilidade. A aplicação do método ABE em disciplinas do curso de Odontologia foi percebido de forma positiva pela quase totalidade dos estudantes.

Descritores: Aprendizagem Baseada em Equipes. Metodologias Ativas. Odontologia.
\end{abstract}

\section{INTRODUÇÃO}

A construção do conhecimento vem sendo o foco principal dos cursos de formação contemporâneos devido às contínuas modificações que vem sofrendo a aprendizagem. Atualmente, os estudantes ingressam na universidade com um conjunto de expectativas e hábitos de estudo diferentes daqueles de gerações anteriores. Muitas vezes, os momentos em sala tornam-se um longo período de exaustão, no qual professores e estudantes acabam caindo em uma rotina acadêmica que parece não se renovar ao longo do 
tempo ${ }^{1}$.

Sob esta perspectiva, é essencial a superação da concepção de educação bancária, na qual o professor faz o depósito de conteúdos, ao passo que o estudante é obrigado a memorizá-los, ou da prática licenciosa, de indivíduos entregues a si mesmo e à própria sorte, num vazio de quem faz e desfaz a seu gosto ${ }^{2}$. Para se contrapor aos métodos tradicionais, emergem as metodologias ativas de aprendizagem, que se constituem em métodos educacionais capazes de permitir o engajamento do educando no processo de aprendizagem, colocando-o no controle do seu próprio percurso de construção do conhecimento ${ }^{3}$.

A aprendizagem baseada em equipes (ABE) representa uma dessas metodologias ativas e foi originalmente desenvolvida para cursos de administração nos anos 1970, por Larry Michaelsen, direcionada para turmas numerosas. Procurava-se criar oportunidades e obter os benefícios do trabalho em pequenos grupos de aprendizagem, de modo que fossem formadas equipes de 5 a 7 estudantes, para trabalharem de forma colaborativa no mesmo espaço físico. Em 2001, o governo norte-americano decidiu financiar educadores das ciências da saúde para que incorporassem novas estratégias de ensino e a $\mathrm{ABE}$ foi escolhida para ser disseminada ${ }^{4}$. Esta estratégia instrucional tem demonstrado ser viável e eficaz na educação médica e demais áreas da saúde ${ }^{5,6,7}$.

O mundo do trabalho contemporâneo exige a formação de profissionais com perfil críticoreflexivo e capazes de trabalhar coletivamente. Considerando-se este contexto, a ABE é um método dinâmico, que proporciona um ambiente motivador e cooperativo, onde a produção coletiva é valorizada. Os estudantes se sentem motivados a participar, o que torna o ambiente de educação mais interessante, minimizando o desinteresse pelo aprendizado $^{8}$.

Algumas pesquisas têm indicado que os estudantes de programas de educação em saúde geralmente têm percepções positivas da abordagem da $\mathrm{ABE}^{5,9}$. Entretanto, estas investigações foram aplicadas em estudantes norte-americanos e asiáticos, não tendo sido encontrada na literatura a aplicação do método de avaliação de percepção em estudantes de Odontologia brasileiros. Este estudo teve o objetivo de analisar a percepção de estudantes do curso de Odontologia de uma universidade brasileira sobre a metodologia de ensino-aprendizagem baseada em equipes em dois componentes curriculares, por meio do Instrumento de Avaliação de ABE pelo Estudante (Team-Based Learning Student Assessment Instrument/TBL-SAI).

\section{METODOLOGIA}

Tratou-se de um estudo transversal com amostra de conveniência composta pelos estudantes regularmente matriculados $(n=83) \mathrm{em}$ duas disciplinas (Biossegurança e Ergonomia 2; Patologia Oral), no segundo semestre de 2017, do curso de Odontologia de uma universidade brasileira. A pesquisa foi aprovada pelo Comitê de Ética em Pesquisa Institucional, com o número de processo 81177017.5.0000.5208. Foram incluídos os sujeitos que concordaram em participar, mediante leitura e assinatura do termo de Consentimento Livre e Esclarecido, que tivessem concluído as respectivas disciplinas em turmas nas quais a $\mathrm{ABE}$ foi utilizada. Os estudantes que tiveram reprovação por falta foram excluídos do estudo.

As duas disciplinas estudadas são componentes curriculares do quarto semestre letivo do curso de graduação em Odontologia, as quais empregaram a ABE como metodologia de ensinoaprendizagem. No início do semestre, em cada disciplina, era realizado o acolhimento, apresentada a disciplina com sua metodologia, sendo formadas equipes com 6 a 8 estudantes com perfil heterogêneo, cuja composição permaneceu fixa ao longo do semestre. Há três fases no método 
ABE. Na fase I, conhecida como preparação préclasse, o professor indicava leituras $\mathrm{e}$ disponibilizava um roteiro de estudos com objetivos e metas. Na fase II, cada estudante respondia dez perguntas de múltipla escolha (etapa de garantia do conhecimento individual) e, em seguida, os estudantes eram reunidos em suas respectivas equipes e passavam a responder o mesmo teste em equipe (etapa de garantia do conhecimento em equipes). Cumpridas essas atividades, o professor reunia a turma para a sedimentação do conteúdo (fase III).

Cada participante respondeu de forma individual o TBL-SAI, um questionário autoaplicável, caracterizado por ser uma ferramenta de avaliação projetada especificamente para analisar as percepções dos estudantes sobre a ABE, com 33 perguntas, cujas respostas estão na escala Likert ${ }^{10}$. Este questionário foi validado para a língua portuguesa em estudo prévio ${ }^{11}$. O TBLSAI é composto por três subescalas: (1) responsabilidade, composta por oito itens; (2) preferência por aula expositiva/tradicional ou $\mathrm{ABE}$, contendo 16 itens; e (3) satisfação do estudante, compreendendo nove itens.

Os dados foram digitados em planilha no Excel e analisados descritivamente (médias, medianas, desvio padrão). Para avaliar o grau de consistência interna do questionário foi obtido o Alfa de Cronbach. Para as análises estatísticas inferenciais utilizou-se o programa SPSS, versão 23.

\section{RESULTADOS}

Um total de 82 estudantes respondeu aos formulários eletrônicos, obtendo-se taxa de resposta correspondente a $98,79 \%$ dos 83 alunos matriculados nas disciplinas estudadas. A amostra foi composta por estudantes predominantemente do sexo feminino $(78 \%, n=64)$ em relação ao masculino $(22 \%, \quad \mathrm{n}=18) . \quad \mathrm{A}$ avaliação da confiabilidade (consistência interna) com a aplicação do teste Alfa de Cronbach foi 0,736, que indicou um grau de consistência interna moderada, aceitável.

Os resultados foram distribuídos conforme as subescalas: responsabilidade (tabela 1), preferência do método (tabela 2) e satisfação do estudante em relação ao método $\mathrm{ABE}$ (tabela 3 ).

$\mathrm{Na}$ tabela 1, observa-se que todas as respostas reafirmam positivamente o sentimento de responsabilidade do estudante quando utilizado o método $\mathrm{ABE}$, especialmente quando indagados sobre a contribuição individual para a aprendizagem coletiva. A maioria também afirmou aproveitar melhor o tempo com estudos prévios a fim de melhorar seu rendimento durante as atividades. Ainda que a maioria diga sentir orgulho de sua contribuição do grupo, uma pequena parte não se sente responsável pela aprendizagem dos colegas.

No que se refere à preferência por aula expositiva/tradicional ou pelo método $\mathrm{ABE}$, conforme ilustra a tabela 2 , os resultados indicam que há uma tendência à maior distração, sonolência, tédio e busca por conversas paralelas durante experiência de aula tradicional. Apenas $1,2 \%$ dos estudantes concordou que se distrai em atividades ABE. Outro aspecto relevante foi a concordância dos estudantes em afirmar que o método $\mathrm{ABE}$ lhes ajuda a recordar o assunto presente e as informações passadas, auxiliando no melhor rendimento no momento das avaliações.

A tabela 3 traz as informações relacionadas à satisfação do estudante. Todos os entrevistados discordaram da afirmação de que o método $\mathrm{ABE}$ seria perda de tempo, assim como nenhum afirmou não gostar de trabalhar em equipe. Outro aspecto relevante foi a concordância por mais de $80 \%$ dos estudantes referente às questões como boas experiências e ao prazer e efetividade das atividades e estudos em equipe, assim como a melhoria do rendimento escolar com este método. 
Tabela 1. Respostas referentes à subescala "Responsabilidade" do TBL-SAI

\begin{tabular}{|c|c|c|c|c|c|c|c|c|c|c|}
\hline \multirow{3}{*}{ QUESTÕES } & \multicolumn{10}{|c|}{ RESPOSTA } \\
\hline & \multicolumn{2}{|c|}{$\begin{array}{l}\text { Discorda } \\
\text { completamente }\end{array}$} & \multicolumn{2}{|c|}{ Discorda } & \multicolumn{2}{|c|}{$\begin{array}{l}\text { Nem discorda, } \\
\text { nem concorda }\end{array}$} & \multicolumn{2}{|c|}{ Concorda } & \multicolumn{2}{|c|}{$\begin{array}{c}\text { Concorda } \\
\text { completamente }\end{array}$} \\
\hline & $\mathbf{n}$ & $\%$ & $\mathbf{n}$ & $\%$ & $\mathbf{n}$ & $\%$ & $\mathbf{n}$ & $\%$ & n & $\%$ \\
\hline $\begin{array}{l}\text { P1. Aproveita seu tempo estudando } \\
\text { antes da aula a fim de estar mais } \\
\text { preparado }\end{array}$ & - & - & 3 & 3,7 & 6 & 7,3 & 21 & 25,6 & 52 & 63,4 \\
\hline $\begin{array}{l}\text { P2. Sente que precisa se preparar } \\
\text { para a aula para obter bom } \\
\text { rendimento }\end{array}$ & - & - & 1 & 1,2 & 2 & 2,4 & 12 & 14,6 & 67 & 81,7 \\
\hline $\begin{array}{l}\text { P3. Contribui para a aprendizagem } \\
\text { dos membros da sua equipe }\end{array}$ & - & - & - & - & 4 & 4,9 & 28 & 34,1 & 50 & 61,0 \\
\hline $\begin{array}{l}\text { P4. Sua contribuição para a equipe } \\
\text { não é importante }\end{array}$ & 68 & 82,9 & 11 & 13,4 & 3 & 3,7 & - & - & - & - \\
\hline $\begin{array}{l}\text { P5. Os membros da sua equipe } \\
\text { esperam que você os ajude em sua } \\
\text { aprendizagem? }\end{array}$ & - & - & 1 & 1,2 & 16 & 19,5 & 30 & 36,6 & 35 & 42,7 \\
\hline $\begin{array}{l}\text { P6. Você é responsável pela } \\
\text { aprendizagem do seu grupo }\end{array}$ & 2 & 2,4 & 13 & 15,9 & 18 & 22,0 & 29 & 35,4 & 20 & 24,4 \\
\hline $\begin{array}{l}\text { P7. Tem orgulho da sua habilidade } \\
\text { em ajudar o seu grupo em sua } \\
\text { aprendizagem }\end{array}$ & 1 & 1,2 & - & - & 19 & 23,2 & 29 & 35,4 & 33 & 40,2 \\
\hline $\begin{array}{l}\text { P8. Tem necessidade em contribuir } \\
\text { para a aprendizagem da sua equipe }\end{array}$ & - & - & 3 & 3,7 & 10 & 12,2 & 31 & 37,8 & 38 & 46,3 \\
\hline
\end{tabular}


Aprendizagem Baseada em Equipes no Ensino odontológico: o que pensam os estudantes?

Tabela 2. Respostas referentes à subescala "Preferência por aula expositiva/tradicional ou ABE" do TBL-SAI

\begin{tabular}{|c|c|c|c|c|c|c|c|c|c|c|}
\hline \multirow{3}{*}{ QUESTÕES } & \multicolumn{10}{|c|}{ RESPOSTA } \\
\hline & \multicolumn{2}{|c|}{$\begin{array}{c}\text { Discorda } \\
\text { completamente }\end{array}$} & \multicolumn{2}{|c|}{ Discorda } & \multicolumn{2}{|c|}{$\begin{array}{l}\text { Nem discorda, } \\
\text { nem concorda }\end{array}$} & \multicolumn{2}{|c|}{ Concorda } & \multicolumn{2}{|c|}{$\begin{array}{c}\text { Concorda } \\
\text { completamente }\end{array}$} \\
\hline & n & $\%$ & $\mathbf{n}$ & $\%$ & $\mathbf{n}$ & $\%$ & $\mathbf{n}$ & $\%$ & $\mathbf{n}$ & $\%$ \\
\hline $\begin{array}{l}\text { P9. Durante uma aula tradicional, você } \\
\text { quase sempre se pega pensando em } \\
\text { coisas não relacionadas à aula }\end{array}$ & 7 & 8,5 & 20 & 24,4 & 13 & 15,9 & 23 & 28,0 & 19 & 23,2 \\
\hline $\begin{array}{l}\text { P10. Se distrai facilmente durante uma } \\
\text { aula tradicional }\end{array}$ & 5 & 6,1 & 16 & 19,5 & 10 & 12,2 & 28 & 34,1 & 23 & 28,0 \\
\hline $\begin{array}{l}\text { P11. Se distrai facilmente durante as } \\
\text { atividades de aprendizagem baseadas em } \\
\text { equipes }\end{array}$ & 40 & 48,8 & 35 & 42,7 & 6 & 7,3 & 1 & 1,2 & - & - \\
\hline $\begin{array}{l}\text { P12. É mais fácil você pegar no sono } \\
\text { durante uma aula expositiva que durante } \\
\text { aulas que usam as atividades de } \\
\text { aprendizagem baseadas em equipes }\end{array}$ & 1 & 1,2 & 4 & 4,9 & 2 & 2,4 & 30 & 36,6 & 45 & 54,9 \\
\hline $\begin{array}{l}\text { P13. Fica entediado durante as atividades } \\
\text { de aprendizagem baseadas em equipes }\end{array}$ & 40 & 48,8 & 33 & 40,2 & 7 & 8,5 & 2 & 2,4 & - & - \\
\hline $\begin{array}{l}\text { P14. Fala sobre coisas não relacionadas à } \\
\text { aula durante as atividades de } \\
\text { aprendizagem baseadas em equipes }\end{array}$ & 27 & 32,9 & 33 & 40,2 & 11 & 13,4 & 9 & 11,0 & 2 & 2,4 \\
\hline $\begin{array}{l}\text { P15. Se lembra facilmente do que é } \\
\text { aprendido quando trabalha em equipe }\end{array}$ & - & - & 3 & 3,7 & 9 & 11,0 & 33 & 40,2 & 37 & 45,1 \\
\hline $\begin{array}{l}\text { P16. Se lembra melhor do assunto } \\
\text { quando o professor faz uma palestra } \\
\text { sobre ele }\end{array}$ & 6 & 7,3 & 7 & 8,5 & 18 & 22,0 & 29 & 35,4 & 22 & 26,8 \\
\hline $\begin{array}{l}\text { P17. As atividades de aprendizagem } \\
\text { baseadas em equipes o ajudam a recordar } \\
\text { de informações passadas }\end{array}$ & - & - & - & - & 5 & 6,1 & 29 & 35,4 & 48 & 58,5 \\
\hline
\end{tabular}


P18. É mais fácil estudar para os testes quando o professor já fez uma palestra sobre o assunto

P19. Se lembra das informações por mais tempo quando realiza/participa das atividades: teste individual + teste em equipe + discussão em grupo + apelação + explicação do professor P20. Se lembra melhor do assunto depois da prática realizada em sala de aula na aprendizagem baseada em equipes

P21. Consegue se lembrar facilmente dos assuntos ministrados nas palestras P22. Depois de discutir com os integrantes da sua equipe, sente dificuldades de se lembrar o que nós discutimos durante a aula

P23. Se sai melhor nas provas quando usam a aprendizagem baseada em equipes dos assuntos a serem

(n)

estudados

P24. Após assistir à palestra, tem dificuldades em se lembrar do que o professor falou durante a aula

\begin{tabular}{|c|c|c|c|c|c|c|c|c|c|}
\hline 1 & 1,2 & 5 & 6,1 & 18 & 22,0 & 27 & 32,9 & 31 & 37,8 \\
\hline- & - & 1 & 1,2 & 5 & 6,1 & 16 & 19,5 & 60 & 73,2 \\
\hline- & - & - & - & 2 & 2,4 & 27 & 32,9 & 53 & 64,6 \\
\hline 1 & 1,2 & 9 & 11,0 & 24 & 29,3 & 37 & 45,1 & 11 & 13,4 \\
\hline 28 & 34,1 & 45 & 54,9 & 8 & 9,8 & 1 & 1,2 & - & - \\
\hline- & - & 4 & 4,9 & 13 & 15,9 & 33 & 40,2 & 32 & 39,0 \\
\hline 15 & 18,3 & 37 & 45,1 & 15 & 18,3 & 13 & 15,9 & 2 & 2,4 \\
\hline
\end{tabular}


Tabela 3. Respostas referentes à subescala "Satisfação do estudante" do TBL-SAI

\begin{tabular}{|c|c|c|c|c|c|c|c|c|c|c|}
\hline \multirow{3}{*}{ QUESTÕES } & \multicolumn{10}{|c|}{ RESPOSTA } \\
\hline & \multicolumn{2}{|c|}{$\begin{array}{c}\text { Discorda } \\
\text { completamente }\end{array}$} & \multicolumn{2}{|c|}{ Discorda } & \multicolumn{2}{|c|}{$\begin{array}{l}\text { Nem discorda, } \\
\text { nem concorda }\end{array}$} & \multicolumn{2}{|c|}{ Concorda } & \multicolumn{2}{|c|}{$\begin{array}{c}\text { Concorda } \\
\text { completamente }\end{array}$} \\
\hline & n & $\%$ & $\mathbf{n}$ & $\%$ & n & $\%$ & $\mathbf{n}$ & $\%$ & n & $\%$ \\
\hline $\begin{array}{l}\text { P25. Gosta das atividades de } \\
\text { aprendizagem baseada em equipes }\end{array}$ & 1 & 1,2 & - & - & 1 & 1,2 & 31 & 37,8 & 49 & 59,8 \\
\hline $\begin{array}{l}\text { P26. Aprende melhor quando está } \\
\text { em grupo }\end{array}$ & 1 & 1,2 & 2 & 2,4 & 11 & 13,4 & 28 & 34,1 & 40 & 48,8 \\
\hline $\begin{array}{l}\text { P27. Acha que a aprendizagem } \\
\text { baseada em equipes representa uma } \\
\text { abordagem efetiva para se aprender }\end{array}$ & - & - & 1 & 1,2 & 4 & 4,9 & 20 & 24,4 & 57 & 69,5 \\
\hline $\begin{array}{l}\text { P28. Não gosta de trabalhar em } \\
\text { equipes }\end{array}$ & 38 & 46,3 & 38 & 46,3 & 6 & 7,3 & - & - & - & - \\
\hline $\begin{array}{l}\text { P29. As atividades de uma aula } \\
\text { baseada em equipes são divertidas }\end{array}$ & - & - & 1 & 1,2 & 3 & 3,7 & 34 & 41,5 & 44 & 53,7 \\
\hline $\begin{array}{l}\text { P30. As atividades de uma aula } \\
\text { baseada em equipes são uma perda } \\
\text { de tempo }\end{array}$ & 64 & 78,0 & 18 & 22,0 & - & - & - & - & - & - \\
\hline $\begin{array}{l}\text { P31. Acha que a aprendizagem } \\
\text { baseada em equipes o ajudou a } \\
\text { melhorar o seu rendimento escolar }\end{array}$ & 1 & 1,2 & 2 & 2,4 & 13 & 15,9 & 31 & 37,8 & 35 & 42,7 \\
\hline $\begin{array}{l}\text { P32. Tem uma atitude positiva em } \\
\text { relação às atividades de } \\
\text { aprendizagem baseadas em equipes }\end{array}$ & - & - & 1 & 1,2 & 2 & 2,4 & 30 & 36,6 & 49 & 59,8 \\
\hline $\begin{array}{l}\text { P33. Teve uma boa experiência } \\
\text { com a aprendizagem baseada em } \\
\text { equipes }\end{array}$ & - & - & 1 & 1,2 & 5 & 6,1 & 28 & 34,1 & 48 & 58,5 \\
\hline
\end{tabular}




\section{DISCUSSÃO}

A alta adesão dos estudantes ao questionário $(98,79 \%)$ mostrou, de acordo com a análise dos resultados, o impacto positivo do método $\mathrm{ABE}$ nos entrevistados. Esta expressão pode ser constatada pelos escores positivos obtidos nas 3 subescalas em que os resultados foram apresentados: sentimento de responsabilidade; preferência pelo método e satisfação.

Outro aspecto importante neste estudo é a maioria de estudantes mulheres. Nas universidades públicas federais, estaduais e nas faculdades privadas, o número de estudantes mulheres ainda é menor do que os de homens, embora a presença feminina no ensino superior esteja aumentando gradativamente e dados quantitativos mais paritários entre os sexos estão sendo observados. No presente estudo, o número de mulheres foi muito superior quando comparado aos homens, $78 \%$ e $22 \%$ respectivamente, o que indica que o alcance do gênero feminino ao ensino superior pode contribuir para uma revisão mais ampla dos papéis de gênero da sociedade. Resultados similares também foram encontrados em um estudo envolvendo a metodologia ABE com estudantes de Medicina ${ }^{13}$.

Durante a formação acadêmica, é desejável que os estudantes experienciem atividades coletivas que exercitem colaboração e cooperação. Especialmente na área de saúde, a perspectiva interprofissional vem sendo requisitada para profissionais que buscam inserção no mundo profissional ${ }^{14}$, os quais necessitam demonstrar habilidades com trabalho em equipe, que se constitui em um dos pilares do método ABE. Conforme as diretrizes curriculares nacionais dos cursos de graduação em odontologia: "o egresso deve estar apto à atuação em equipes interprofissionais, interdisciplinares e transdisciplinares ${ }^{15}$."

Nesta investigação, os estudantes destacaram os benefícios do trabalho em equipe e a importância da sua contribuição individual para os resultados positivos em grupo. Experiências semelhantes também podem ser encontradas em outros estudos ${ }^{8,16}$. No caso particular dos futuros profissionais da área saúde, é premissa do Sistema Único de Saúde (SUS) a atuação interprofissional baseada no trabalho em equipe, o que nos mostra que o método $\mathrm{ABE}$ tem um potencial muito maior de preparar os estudantes para os desafios profissionais do século XXI quando comparado ao modelo de ensino tradicional, onde há uma distribuição de conhecimentos unidirecional de um docente para os discentes ${ }^{2}$.

Estudos mostram que não só as metodologias ativas de ensino-aprendizagem estimulam o trabalho em equipe, como também oferecem uma resposta positiva dos estudantes sobre esta experiência. Um estudo utilizando metodologias ativas com a disciplina eletiva de Políticas Públicas em Saúde Mental na Universidade Federal do Rio de Janeiro (UFRJ), com estudantes de Medicina e Psicologia, obteve como resposta satisfação de $60 \%$ em trabalhos com pequenos grupos, principalmente com esta ferramenta como auxílio para melhor aprendizagem ${ }^{17}$. No presente estudo, tendo a ABE como uma metodologia ativa, os estudantes responderam ter orgulho da sua habilidade de ajudar seu grupo na aprendizagem $(75,6 \%)$. Também responderam ter a necessidade de contribuir para a sua equipe $(84,1 \%)$, mostrando que o trabalho em equipe é bem aceito pela maioria dos estudantes.

Outro aspecto relevante do método $\mathrm{ABE}$ faz menção ao protagonismo do estudante na busca do conhecimento, assumindo um papel cada vez mais ativo, descondicionando-se da 
atitude de mero receptor de conteúdos, buscando efetivamente conhecimentos relevantes aos problemas e aos objetivos da aprendizagem ${ }^{2}$. Neste estudo, os estudantes afirmaram se sentir mais responsáveis com o método $\mathrm{ABE}$, buscando o estudo prévio para a realização das atividades avaliativas nas aulas com o objetivo de melhorarem seus resultados (89\%). Outros autores também encontraram que o método ABE estimula os estudantes a apresentarem uma postura mais ativa na busca do conhecimento, obtendo um maior envolvimento com a referida metodologia, corroborando com os nossos resultados ${ }^{6,18,19}$.

Estudo em uma faculdade brasileira de Medicina concluiu que os estudantes demonstraram sua preferência pela $\mathrm{ABE}$ quando comparada ao modelo tradicional de aula, relatando que esta metodologia de ensinoaprendizagem torna as aulas mais dinâmicas, participativas, produtivas e estimula uma maior frequência de estudo e interação no grupo para resolverem as atividades propostas ${ }^{8}$. De igual maneira, nos resultados do questionário aplicado nesta pesquisa, os estudantes referiram que no método $\mathrm{ABE}$ é mais difícil haver distração, sonolência ou tédio nas aulas, além do trabalho em equipe facilitar a lembrança do assunto ministrado, consequentemente levando a melhores resultados nas provas.

As propostas educativas tradicionais, as quais se embasam na transmissão centrada no papel do professor, vem constantemente sendo desafiadas por propostas pedagógicas inovadoras. Esta necessidade para a transformação nos métodos de ensinoaprendizagem pode ser observada nesta investigação, uma vez que $97,6 \%$ dos estudantes responderam que concordam e concordam totalmente que gostam das atividades da $\mathrm{ABE}$, assim como $92,6 \%$ dos mesmos relataram ter uma boa experiência com o método $\mathrm{ABE}$. Os dados demonstram a fácil adesão ao método $\mathrm{ABE}$ pelos estudantes, tal característica também é encontrada em outros estudos $6,16,19,20$. Estes resultados confirmam a necessidade de uma substituição progressiva das metodologias de ensino-aprendizagem conservadoras por metodologias ativas, colocando o estudante como protagonista da construção dos seus saberes e o docente como ponte para estes saberes, porém não um fim em seu meio ${ }^{2,8}$.

Foi relatado pelos estudantes que a efetividade das atividades nas aulas mais o trabalho em equipes do método $\mathrm{ABE}$ ajudou a melhorar o seu rendimento escolar. Outros estudos utilizando o método $\mathrm{ABE}$ com estudantes de Fisioterapia, Medicina e Odontologia mostraram maiores notas dos alunos quando comparado ao modelo de aula tradicional $^{6,13,18}$. Nesta investigação, 79,2\% dos estudantes relataram se sair melhor nas provas quando utilizaram a $\mathrm{ABE}$ nos assuntos a serem abordados, assim como 93,4\% dos mesmos afirmaram que as atividades da $\mathrm{ABE}$ os ajudam a recordar as informações obtidas em aula. O melhor aproveitamento escolar por parte dos estudantes no método ABE aponta que metodologias ativas são mais eficazes para a aprendizagem e fixação dos conhecimentos abordados. O método ABE, quando utilizado em um módulo de prótese fixa para estudantes de Odontologia em uma universidade do Japão, se mostrou mais eficiente no ensino quando comparado aos métodos tradicionais conservadores ${ }^{7}$.

$\mathrm{O}$ presente estudo ainda mostrou que a grande maioria dos estudantes, mais de $80 \%$, reconheceu a importância da $\mathrm{ABE}$ por meio de respostas extremamente positivas sobre a própria melhora na aprendizagem quando realizada em grupo, sua efetividade e melhoria do rendimento escolar, além do que nenhum estudante respondeu que a referida metodologia é perda de 
tempo. Resultados similares também foram encontrados em outros estudos 6,7,13,16,18-22.

Ficou evidente que o método $\mathrm{ABE}$ se apresentou como uma promissora alternativa de mudança do modelo de ensino conservador vigente na área da saúde. No entanto, é necessário destacar a necessidade de ampliação do quantitativo amostral, o que nesta pesquisa não foi possível, pelo fato de o referido curso de Odontologia possuir apenas duas disciplinas que utilizam esta metodologia. Além disso, aponta-se a necessidade de desenvolvimento de estudos comparativos sobre o desempenho dos estudantes com o método $\mathrm{ABE}$ em relação ao modelo tradicional de aprendizagem.

\section{CONCLUSÃO}

$\mathrm{O}$ método $\mathrm{ABE}$ foi percebido de forma positiva pela quase totalidade dos estudantes entrevistados, configurando-se como uma estratégia de ensino-aprendizagem a ser considerada no planejamento pedagógico dos componentes curriculares dos cursos de Odontologia.

\section{ABSTRACT \\ Team-Based Learning in Dentistry teaching: what do students think?}

Team-based learning (TBL) is a dynamic method that provides a motivating and cooperative environment, with appreciation of collective production. The aim of this paper was to analyze the perception of undergraduate dentistry students of a Brazilian university on the TBL method. This is a cross-sectional study with convenience sample $(n=83)$, in which each student answered a self-administered questionnaire - Student's TBL Evaluation Instrument. Data were analyzed based on descriptive statistics. Moderate, acceptable internal consistency $($ Cronbach's alpha $=0.736$ ) was found. The means of answers obtained indicated that students showed preference and satisfaction regarding the use of the TBL method, being able to arouse greater sense of responsibility. The application of the TBL method was positively perceived by almost all dentistry students.

Descriptors: Team Based Learning. Active Methodologies. Dentistry.

\section{REFERÊNCIAS}

1. Michaelsen LK, Davidson N, Major $\mathrm{CH}$. Team-Based Learning Practices and Principles in Comparison with Cooperative Learning and Problem-Based Learning. J Excell Coll Teach. 2014;25:54-84.

2. Mitre SM, Siqueira-Batista R, Girardi-deMendonça JM, Morais-Pinto NM, Meirelles $\mathrm{CAB}$, Pinto-Porto $\mathrm{C}$, et al. Metodologias ativas de ensino-aprendizagem na formação profissional em saúde: debates atuais. Ciênc Saúde Colet. 2008;13:2133-44.

3. Prince M. Does active learning work? A review of the research. $\mathrm{J}$ Eng Educ. 2004;93(3):223-1.

4. Bollela VR, Senger MH, Tourinho FCV, Amaral E. Aprendizagem baseada em equipes: da teoria à prática. Med. 2014;47(3):293-300.

5. Abdelkhalek $\mathrm{N}$, Hussein $\mathrm{A}$, Gibbs $\mathrm{T}$, Hamdy H. Using team-based learning to prepare medical students for future problembased learning. Med Teach. 2010;32(2):1239.

6. Livingston B, Lundy $\mathrm{M}$, Harrington $\mathrm{S}$. Physical therapy students' perceptions of team-based learning in gross anatomy using the Team-Based Learning Student Assessment Instrument. J Educ Eval Health Prof. 2014;11:1.

7. Takeuchi H, Omoto K, Okura K, Tajima T, Suzuki Y, Hosoki M et al. Effects of teambased learning on fixed prosthodontic education in a Japanese School of Dentistry. J Dent Educ. 2015;79(4):417-23.

8. Bandeira DMA, Silva MA, Vilela RQB. 
Aprendizagem Baseada em Equipe. Saúde Sociedade. 2017;2(1):371-9.

9. Addo-Atuah J. Performance and perceptions of pharmacy students using team-based learning (TBL) within a global health course. Inov Pharm. 2011;2(2):1-16.

10. Mennenga HA. Development and Psychometric Testing of the Team-Based Learning Student Assessment Instrument. Nurse Educ. 2012;37(4):168-72.

11. Souza FB, Carvalho EJA, Castro BL, Matos EMO, Arreguy IMS, Figueiredo-Filho AO et al. Team-based learning student assessment instrument in Brazilian dental education: a validation study. J Dent Health Oral Disord Ther. 2019;10(3):182-6.

12. Barreto A. A mulher no ensino superior: Distribuição e representatividade. Cad GEA. 2014;1(6):3-46.

13. Koles PG, Stolfi A, Borges NJ, Nelson S, Parmelee DX. The impact of team-based learning on medical students' academic performance. Acad Med. 2010; 85(11):1739-45.

14. Orsini CA, Danús MT, Tricio JA. La importancia de la educación interprofesional en la enseñanza de la odontología: una revisión sistemática exploratoria analizando el dónde, el porqué y el cómo. Educ Med. 2018; 20(S1):152-64.

15. Brasil. Resolução $\mathrm{n}^{\circ}$ 03/2002 de 19 de fevereiro de 2002. Dispõe sobre as Diretrizes Curriculares Nacionais dos cursos de graduação em odontologia. Diário Oficial da União 2002. [Acesso em 13 dez. 2018]. Disponível em: http://portal.mec.gov.br/cne/ arquivos/pdf/CES032002.pdf.

16. Pogge E. Evaluation of an interprofessional team-based learning nutrition and lifestyle modification course. J Interprof Care.
2016;30(2):248-50.

17. Gomes MPC, Ribeiro VMB, Monteiro DM, Leher EMT, Louzada RCR. O uso de metodologias ativas no ensino de graduação nas ciências sociais e da saúde: avaliação dos estudantes. Ciênc Educ. 2010;16(1):181-98.

18. Haj-Ali R, Al Quran F. Team-based learning in a preclinical removable denture prosthesis module in a United Arab Emirates dental school. J Dent Educ. 2013;77(3): 351-7.

19. Simonson SR. Making students do the thinking: team-based learning in a laboratory course. Adv Physiol Educ. 2014;38(1):4955.

20. Akbary M, Kargozar S. Team based learning; An innovative teaching method in preclinic course of restorative dentistry and its impact on the students' clinical skills, Mashhad dental school. J Dent Med. 2016;29(2):136-43.

21. Mattheos N, Storrs M, Foster L, Oberholzer T. Intraprofessional, team-based treatment planning for oral health students in the comprehensive care clinic. J Dent Educ. 2012;76(12):1589-99.

22. Keshani F, Alikhasi M, Azmoodeh, F. Comparison of the effects of lecture and Team Based Learning (TBL) on oral and maxillofacial pathology learning in dental students. J Qazvin Univ Med Sci. 2015;19(3):60-3.

\section{Correspondência para:}

Elaine Judite de Amorim Carvalho

e-mail: elaine_judite@yahoo.es

Departamento de Clínica e Odontologia, UFPE

Av. Prof. Artur de Sá, 329-481

Cidade Universitária

52171-011 Recife/PE 